\title{
Adherence to National Guidelines for Drug Treatment of Suspected Acute Myocardial Infarction
}

\section{Citation}

McLaughlin, Thomas J. 1996. "Adherence to National Guidelines for Drug Treatment of Suspected Acute Myocardial Infarction." Archives of Internal Medicine 156 (7) (April 8): 799. doi:10.1001/archinte.1996.00440070131015.

\section{Published Version}

doi:10.1001/archinte.1996.00440070131015

\section{Permanent link}

http://nrs.harvard.edu/urn-3:HUL.InstRepos:32696165

\section{Terms of Use}

This article was downloaded from Harvard University's DASH repository, and is made available under the terms and conditions applicable to Other Posted Material, as set forth at http:// nrs.harvard.edu/urn-3:HUL.InstRepos:dash.current.terms-of-use\#LAA

\section{Share Your Story}

The Harvard community has made this article openly available.

Please share how this access benefits you. Submit a story.

\section{Accessibility}




\title{
Adherence to National Guidelines for Drug Treatment of Suspected Acute Myocardial Infarction
}

\author{
Evidence for Undertreatment in Women and the Elderly
}

Thomas J. McLaughlin, ScD; Stephen B. Soumerai, ScD; Donald J. Willison, ScD; Jerry H. Gurwitz, MD; Catherine Borbas, PhD; Edward Guadagnoli, PhD; Barbara McLaughlin; Nora Morris, MA; Su Chun Cheng, ScD; Paul J. Hauptman, MD; Elliott Antman, MD; Linda Casey, MS; Richard Asinger, MD; Fredarick Gobel, MD

Background: Evidence-based guidelines for the treatment of patients with acute myocardial infarction (AMI) have been published and disseminated by the American College of Cardiology and the American Heart Association. Few studies have examined the rates of adherence to these guidelines in eligible populations and the influence of age and gender on highly effective AMI treatments in community hospital settings.

Mothodsz Medical records of 2409 individuals admitted to 37 Minnesota hospitals between October 1992 and July 1993 for AMI, suspected AMI, or rule-out AMI, and meeting electrocardiographic, laboratory, and clinical criteria suggestive of $A M I$ were reviewed to determine the proportion of eligible patients who received thrombolytic, $\beta$-blocker, aspirin, and lidocaine hydrochloride therapy. The effects of patient age, gender, and hospital teaching status on the use of these treatments were estimated using logistic regression models.

Results: Eligibility for treatment ranged from $68 \%$ $(n=1627)$ for aspirin therapy, $38 \%(n=906)$ for lidocaine therapy, and $30 \%(n=734)$ for thrombolytic therapy to $19 \%(n=447)$ for $\beta$-blocker therapy. Seventy-two per- cent of patients eligible to receive a thrombolytic agent received this therapy; $53 \%$ received $\beta$-blockers; $81 \%$ received aspirin; and $88 \%$ received lidocaine. Among patients ineligible for lidocaine therapy $(n=1503), 20 \%$ received this agent. Use of study drugs was lower among eligible elderly patients, especially those older than 74 years (thrombolytic agent: odds ratio, $0.2 ; 95 \%$ confidence interval, 0.1 to 0.4 ; aspirin: odds ratio, $0.4,95 \%$ confidence interval, 0.3 to $0.6 ; \beta$-blocker: odds ratio, 0.4 ; $95 \%$ confidence interval, 0.2 to 0.8 ). Female gender was associated with lower levels of aspirin use among eligible patients (odds ratio, $0.7 ; 95 \%$ confidence interval, 0.6 to 0.9 ); and there was a trend toward lower levels of $\beta$-blocker and thrombolytic use among eligible women.

Conclusions: Use of lifesaving therapies for eligible patients with AMI is higher than previously reported, particularly for aspirin and thrombolytic use in nonelderly patients. Lidocaine is still used inappropriately in a substantial proportion of patients with AMI. Increased adherence to AMI treatment guidelines is required for elderly patients and women.

(Arch Intern Med. 1996;156:799-805)
The affiliations of the authors appear in the acknowledgment section at the end of the article.

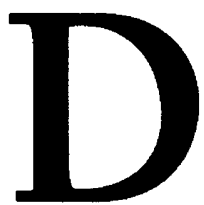
of death in the United States. ${ }^{1}$ Randomized controlled trials ${ }^{2-7}$ of patients with AMI provide strong and consistent evidence that early administration of several drug therapies, including thrombolytic agents, aspirin, and $\beta$-blockers, substantially reduce mortality and other adverse events. Recent data ${ }^{8}$ also indicate that other common AMI treatments, such as prophylactic use of lidocaine hydrochloride, which reduces the likelihood of primary ventricular fibrillation, may lead to increased mortality, especially in uncomplicated AMI. Nevertheless, lidocaine continues to be used in patients for whom there is no evidence of proven benefit. $\mathrm{Na}$ tional evidence-based guidelines for the early management of patients with AMI have been published and disseminated. ${ }^{9}$ However, a critical review ${ }^{10}$ of welldesigned studies, which examined the effectiveness of interventions aimed at improving drug-prescribing behaviors, has shown that the dissemination of printed materials alone appears ineffective in changing physicians' clinical practice, sug-, gesting that adoption of practice recommendations may be suboptimal.

Earlier studies ${ }^{11-14}$ of drug treatment

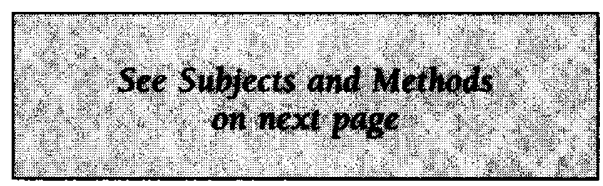




\section{SUBJECTS AND METHODS}

\section{SETTING AND STUDY HOSPITALS}

This investigation involved collaboration between the Boston-based research team and the Minnesota Clinical Comparison and Assessment Program (MCCAP), a quality improvement program of the Healthcare Education and Research Foundation in St Paul. ${ }^{17}$ At the time this study began, MCCAP had obtained the active participation of 45 hospitals throughout the state, representing about $60 \%$ of statewide admissions and $55 \%$ of all AMI cases. Based on published reports of randomized controlled trials, guidelines for study drugs were developed by the American College of Cardiology and the American Heart Association and disseminated to Minnesota hospitals by mail.

Thirty-seven of the hospitals participating in the MCCAP, representing a broad cross section of urban and nonurban hospitals ( $54 \%$ and $46 \%$, respectively), and a wide range of bed sizes, chose to enroll in this study. Nineteen (51\%) study hospitals had fewer than 100 staffed beds; 16 (43\%) had 100 to 500 staffed beds; and two (5\%) had 500 or more staffed beds. Two hospitals represented academic medical centers; the remainder were urban, suburban, or rural community hospitals. The majority of hospitals in the MCCAP that did not participate in the study treated very small numbers of patients with AMI or were participating in other studies.

\section{CRITERIA FOR IDENTIFYING STUDY POPULATION}

This investigation measured consistency of physician prescribing with guideline recommendations during the acute phase of illness. Patients were identified through medical chart review of diagnoses and clinical and laboratory findings at admission rather than at discharge. This avoided the problem of evaluating care for patients presenting atypically and often not diagnosed with AMI until later in the hospital stay.
The pool available for screening included 4968 patients with a diagnosis of AMI, suspected AMI, or rule-out AMI admitted to the 37 study hospitals during the study period. Patients were included in the study if they met at least two of the following criteria for AMI: (1) an electrocardiogram (ECG) with positive findings (defined as physician documentation that ECG was consistent with AMI (ie, Q-wave or T-wave inversion, or ST-segment depression or elevation $\geq 1 \mathrm{~mm}$ ); (2) positive enzyme evidence (elevation of the isoenzyme muscle-brain [MB] subfraction of creatine kinase); or (3) clinical symptoms (arm or shoulder pain, chest pain, diaphoresis, dyspnea, nausea or vomiting, or neck/jaw pain consistent with cardiac ischemia). Patients were excluded from the study if they were dead on arrival, were transferred from a nonstudy hospital, or had suffered an AMI 2 weeks before the index admission.

\section{INDICATIONS AND CONTRAINDICATIONS FOR STUDY DRUGS}

All absolute and relative contraindications as well as indications for use of thrombolytic agents, aspirin, $\beta$-blockers, and lidocaine were summarized by a panel of cardiologists and internists on the research team (Figure 1). This involved review of all clinical trials of target therapies ${ }^{3}$ to determine eligible populations for the study drugs and identification of all indications and contraindications from the most recent (1990) AMI practice guidelines by the American College of Cardiology and the American Heart Association. ${ }^{9}$ Important data on clinical variables for the indication of thrombolytic agents (ST-segment elevation, time from symptom onset to presentation) or of lidocaine (sustained [ $30 \mathrm{sec}$ onds of $>100 \mathrm{bpm}$ ] or nonsustained ventricular tachycardia, ventricular fibrillation, or frequent $[>6$ minutes] premature ventricular contractions) were present for more than $98 \%$ of all patients. Because the treatment recommendation for lidocaine is controversial, we used a liberal definition of eligibility in accord with guidelines by the American College of Cardiology and the American Heart Association (Figure 1). of AMI based on administrative claims or registry data examined gross use of effective AMI therapies. In these studies, however, information on indications or contraindications to therapy was limited; therefore, patient eligibility for specific AMI therapies could not be established. Two recent reports, ${ }^{15,16}$ however, did establish eligibility but only for the elderly, who make up only about half of all patients with AMI. Because of these limitations, levels of appropriate use of thrombolytic agents, aspirin, $\beta$-blockers, and other lifesaving therapies may have been underestimated, and the quality of AMI pharmacotherapy in large samples of patients of all ages in community hospitals remains unknown.

This study reports rates of adherence to consensus guideline recommendations for aspirin, $\beta$-blocking agents, lidocaine, and thrombolytic agents among $2409 \mathrm{pa}$ tients with AMI treated at 37 Minnesota hospitals from October 1992 to July 1993. We focused on physician de- cision making in the context of suspected or confirmed AMI immediately after presentation (intention to treat). In addition, we determined whether patient age, gender, hospital location (urban vs nonurban), or hospital teaching status was related to rates of adherence to the published practice guidelines.

RISI I I

\section{PATIENT SAMPLE}

Among 4968 patients admitted for suspected AMI, 48\% $(n=2409)$ met criteria for study inclusion. The median and mean numbers of study patients per hospital were 42 and 65 , respectively. The baseline characteristics of study patients are presented in Toble $\mathbf{I}$.

Ninety-two percent of study patients had a discharge diagnosis of AMI, indicating a high predictive value of the algorithm in identifying patients at admis- 


\section{DEVELOPMENT OF ABSTRACTING INSTRUMENT}

The medical record abstraction instrument was designed to retrieve data on demographics; admission and discharge dates; insurance status; inclusion and exclusion criteria; admission data, including initial medical contact, time from first symptoms to hospital presentation; diagnostic impressions; identities of all medications taken before admission; inpatient procedures; ECG evidence of AMI; laboratory evidence of AMI; medical history at admission; clinical findings (symptoms and physical findings) on presentation and during the first 24 hours of hospitalization; and identity and time of administration of all drugs in the first 48 hours of admission, including during emergency transport and in the emergency department. Indications and contraindications for all study drugs were able to be developed from the information in the above categories. To increase sensitivity for several important contraindications (congestive heart failure, uncontrolled hypertension, etc), we abstracted data from both physicians' and nurses' notes.

\section{DATA COLLECTION AND INTEGRITY}

Nurses experienced in the care of patients with AMI performed all data collection for this study. Successful abstractors were required to complete 2 days of training and data collection exercises, and demonstrate initial and ongoing interrater agreement with a criterion review of $95 \%$ or higher. Retrospective audits of a random sample of $10 \%$ of each abstractor's completed cases were conducted to ensure that each abstractor met and maintained the data quality standard of 95\% all-item agreement with the MCCAP auditor. Abstractors falling below this standard after additional training were released, and their charts were reabstracted.

We tested the validity of chart-based ECG information indicating thrombolytic treatment (ST segment, elevation $\geq 1 \mathrm{~mm}$ in two or more contiguous leads, excluding changes consistent with early repolarization or pericarditis; or new or presumably new left bundlebranch block) by collecting the first two ECG tracings for a $25 \%$ random sample of study patients $(n=534)$. Two independent cardiologists blind to the original ECG read- ings reviewed and interpreted ECG tracings of the $25 \%$ random sample. We then calculated the congruence between indication of ST-segment elevations of $1 \mathrm{~mm}$ or more in the medical chart (yes or no) with the cardiologists' reviews of the tracings (yes or no).

\section{DESCRIPTION OF ANALYTICAL MODELS}

For analysis, we defined eligibility as the absence of absolute or relative contraindications to aspirin, $\beta$-blockers, lidocaine, and thrombolytic agents as well as the presence of the appropriate indication (Figure 1). For example, after we excluded patients with medical contraindications, eligibility for use of thrombolytic agents was further restricted to patients presenting within 12 hours of onset of symptoms and with an ST-segment elevation of $1 \mathrm{~mm}$ or more. Adherence to guidelines (yes or no) for use of thrombolytic agents, $\beta$-blockers, and aspirin was defined as receipt of drug by an eligible patient within 24 hours of first contact with the hospital. We calculated observed adherence rates for each medication as the proportion of eligible patients receiving thrombolytic agents, $\beta$-blockers, or aspirin. For the purposes of this study, we defined nonadherence to lidocaine treatment guidelines as use among patients without the previously described indications.

To examine the association of patient and hospital characteristics with guideline adherence rates, we analyzed patient and hospital-level data for each study drug. A logistic regression model was used that controlled for correlation of binary observations (adherence to guidelines, yes or no) within hospitals. ${ }^{18}$ Regression models included terms for patient gender; patient age interval ( $<65,65$ to $74,>74$ years); teaching status of hospital; and urban vs nonurban location of the hospital. Odds ratios (OR) and confidence intervals $(\mathrm{CI})$ were calculated directly from the estimated regression coefficients and their SEs. Adjusted proportions for receipt of the study drugs among eligible patients (or ineligible patients in the case of lidocaine) were estimated using the coefficients of the logistic regression analysis and represent estimated proportions controlling for the presence of all patient and hospital study variables. sion with suspected AMI. To further validate the abstracted ECG information used for identifying ST elevation (indication for thrombolytic therapy), we compared the medical record interpretation of ECG tracings with those performed by research team cardiologists. Among 250 patients with medical record documentation of ST-segment elevation, both cardiologist reviewers agreed with this interpretation in $86 \%$ of cases, a high level of concordance considering that cardiologists had access to only the first two ECG tracings in the medical chart.

\section{OVERALL RATES OF ADHERENCE TO AMI TREATMENT GUIDELINES}

After controlling for all other characteristics in the regression models, the largest gaps in adherence to treatment guidelines among eligible patients were observed for thrombolytic agents and $\beta$-blockers.
Seven hundred thirty-four of the 2409 patients were eligible for thrombolytic therapy, but only $72 \%$ of patients eligible to receive thrombolytic therapy actually received this therapy. Of $447(19 \%)$ of the 2409 study patients eligible to receive $\beta$-blockers, 237 (53\%) received therapy in the first 24 hours. However, the main reasons for ineligibility for these drugs were often transitory conditions such as bradyarrhythmias and hypotension.

Thirty-eight percent of study patients $(n=906)$. had a medical record documentation of a 30-second run of sustained ventricular tachycardia of more than $100 \mathrm{bpm}$, ventricular fibrillation, nonsustained ventricular tachycardia, or frequent premature ventricular contractions; $88 \%$ of these individuals received lidocaine. However, among those with no indication $(n=1503), 300(20 \%)$ received this potentially harmful drug. Aspirin was administered to 1318 (81\%) eligible patients. 


\section{ORAL ASPIRIN}

Indication: All patients with $\mathrm{AM}$ l and without any contraindications.

Absolute Contraindications: History of allergy to aspirin; serious gastrointestinal bleeding-hematemesis; blood in nasogastric aspirate.

Relalive Coniraindications: Asthma (with nasal polyps); history of bleeding/significant risk of bleeding; history of peptic ulcer disease.

\section{B-BLOCKERS}

Indication: All patients with AMI and without any contraindications.

Absolute Contraindications: Bradycardia ( $<60$ beats per minute); low systolic blood pressure ( $<100 \mathrm{~mm} \mathrm{Hg}$ ); severe left ventricular failure (rales greater than $10 \mathrm{~cm}$ from base of lungs [ $10 \mathrm{~cm}=1 / 3$ from base]); severe bronchospastic lung disease; signs of peripheral hypoperfusion; atrioventricular condition abnormalities; history of adverse reaction to $\beta$-blockers.

Relative Contraindications: Systolic blood pressure $<110 \mathrm{~mm} \mathrm{Hg}$; history of asthma; current use of verapamil or diltiazem; severe peripheral vascular disease; difficult-to-control, severe, insulin-dependent diabetes.

\section{THROMBOLYTIC AGENTS}

Indications: All patients with AMI or suspected AMI presenting within 12 hours of onset of symptoms; ST-segment elevation $\geq 1 \mathrm{~mm}$; no medical contraindications.

Absolute Contraindications: Active internal bleeding; suspected aortic dissection; prolonged or traumatic cardiopulmonary resuscitation; intracranial neoplasm; hemorrhagic ophthalmic conditions; pregnancy; previous allergic reaction to the thrombolytic agent; sustained systolic blood pressure $>180 \mathrm{~mm} \mathrm{Hg}$; or diastolic blood pressure $>110 \mathrm{~mm} \mathrm{Hg}$; any recorded blood pressure $>200 / 120 \mathrm{~mm} \mathrm{Hg}$ on admission; trauma or surgery in past 2 weeks; AMI onset $>24$ hours.

Relative Contraindications: Major bleeding; recent trauma or surgery >2 weeks; history of chronic severe hypertension with or without drug therapy history of cerebrovascular accident; current use of warfarin anticoagulants: prior use of streptokinase or APSAC; significant liver dysfunction; active peptic ulcer; AMl onset $>12$ hours.

\section{LIDOCAINE HYOROCHLORIDE}

Indications: Patients with AMI (ACC/AHA class )) or suspected AM (ACC/AHA class Ila) with frequent $(>6)$ ventricular premature beats; nonsustained or sustained ( $>30$ seconds) ventricular tachycardia at a rate $>100 \mathrm{bpm}$; ventricular fibrillation.

Absolute Contraindications: Allergy to lidocaine.

Finure 1. List of indications and contraindications for study drugs. $A M$ indicates acute myocardial infarction; APSAC, anisoylated plasminogen activator complex; and ACC/AHA, American College of Cardiology and the American Heart Association.

\section{EFFECT OF PATIENT AGE ON RATES OF ADHERENCE TO GUIDELINES}

Figure 2 illustrates unadjusted guideline adherence rates for the study drugs by age interval among patients eligible to receive aspirin, $\beta$-blockers, and thrombolytic agents. In general, we observed a trend toward reduced use of study drugs with increasing age.

Table 2 presents adjusted proportions and ORs for receipt of the study drugs by patient age category and other study characteristics, controlling for all other patient and hospital characteristics. For all four drugs, patients aged 75 years or older were significantly less likely to be treated than those aged 64 years or younger, regardless of whether the agent was likely to be beneficial (aspirin, $\beta$-blockers, thrombolytic agents) or ineffective (lidocaine). This

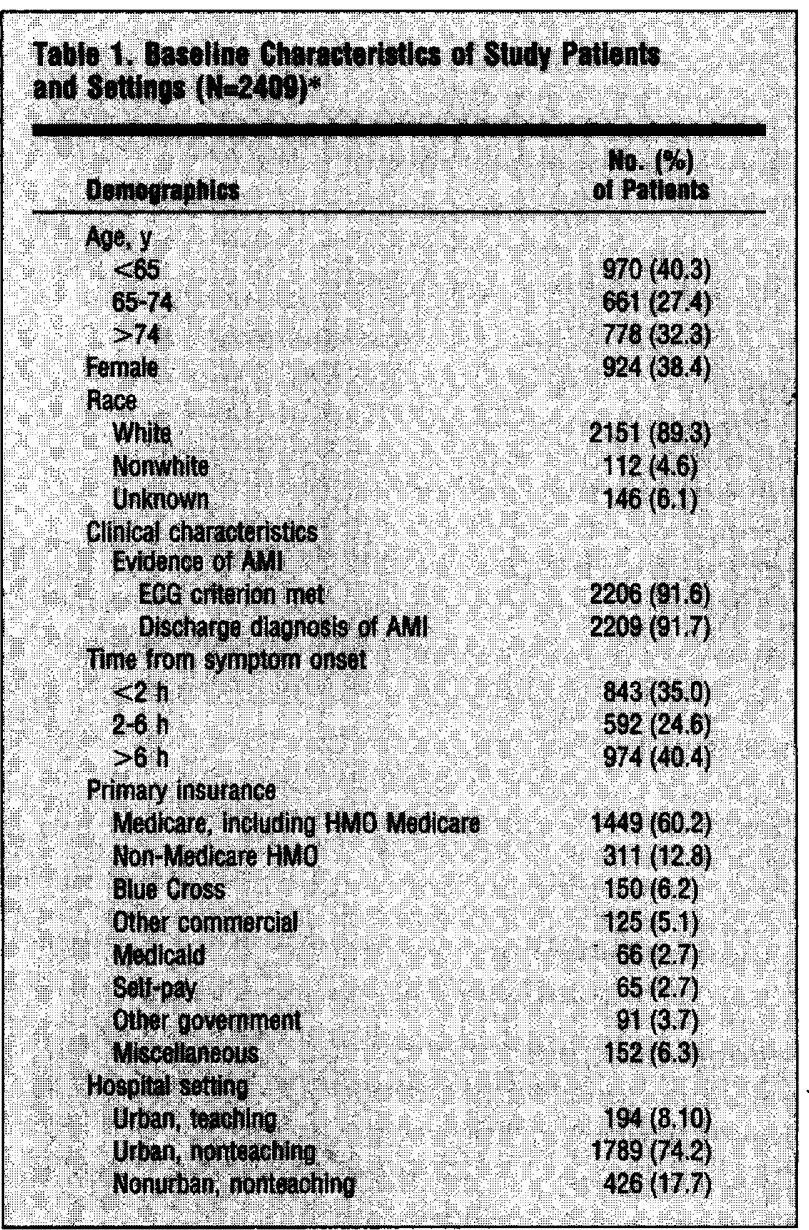

*AMI indicates acute myocardial infarction; ECG, electrocardiogram; and $H M O$, health maintenance organization.

level of undertreatment of those aged 75 years or older was most pronounced for thrombolytic agents (OR, 0.2; $95 \% \mathrm{CI}, 0.1$ to 0.4 ) although significantly lower levels of $\beta$-blockers (OR, $0.4 ; 95 \% \mathrm{CI}, 0.2$ to 0.9 ) and aspirin (OR, $0.4 ; 95 \% \mathrm{CI}, 0.3$ to 0.6 ) were also observed. Inappropriate lidocaine administration was also lower among those aged 75 years or older compared with those younger than 65 years (OR, $0.6 ; 95 \% \mathrm{CI}, 0.6$ to 0.8 ).

We also observed a nonsignificant trend toward lower use of $\beta$-blockers and thrombolytic agents among the "young-old" (aged 65 to 74 years) in comparison with nonelderly patients (estimated proportions of $80 \%$ vs $76 \%$ for thrombolytic agents, $P=.08 ; 60 \%$ vs $51 \%$ for $\beta$-blockers, $P=.33$; Table 2 ). Aspirin use was significantly lower among those aged 65 to 74 years compared with those younger than 65 years (OR, $0.7 ; 95 \%$ $\mathrm{CI}, 0.5$ to 0.9 ).

\section{EFFECT OF PATIENT GENDER ON RATES OF ADHERENCE TO GUIDELINES}

Among patients eligible for treatment, use of all study drugs was consistently lower for women than for men (Figure 3). After adjusting for age and hospital type, women were less likely to be treated with aspirin (OR, $0.7 ; 95 \% \mathrm{CI}, 0.6$ to 0.9 ) and thrombolytic agents (OR, 
$0.7 ; 95 \% \mathrm{CI}, 0.6$ to 1.0 ) than men (Table 2). A similar trend was observed for those treated with $\beta$-blockers and lidocaine (Table 2).

\section{EFFECT OF HOSPITAL TYPE ON RATES OF ADHERENCE TO GUIDELINES}

In general, we did not observe consistent differences in adherence to treatment guidelines for aspirin, $\beta$-blockers, thrombolytic agents, or lidocaine among urban, teaching hospitals, urban, nonteaching hospitals, and nonurban, nonteaching hospitals (Figure 4). After controlling for patient characteristics, the largest effect of hospital type on guideline adherence patterns was for use of lidocaine among patients without indication for this therapy (Table 2). Urban, teaching hospitals were approximately four times less likely than urban, nonteaching to use lidocaine inappropriately (OR, $0.2 ; 95 \% \mathrm{CI}, 0.1$ to 0.6 ).

\section{(ONIINT}

The present study represents the largest investigation to date of rates of adherence to national AMI drug treat-

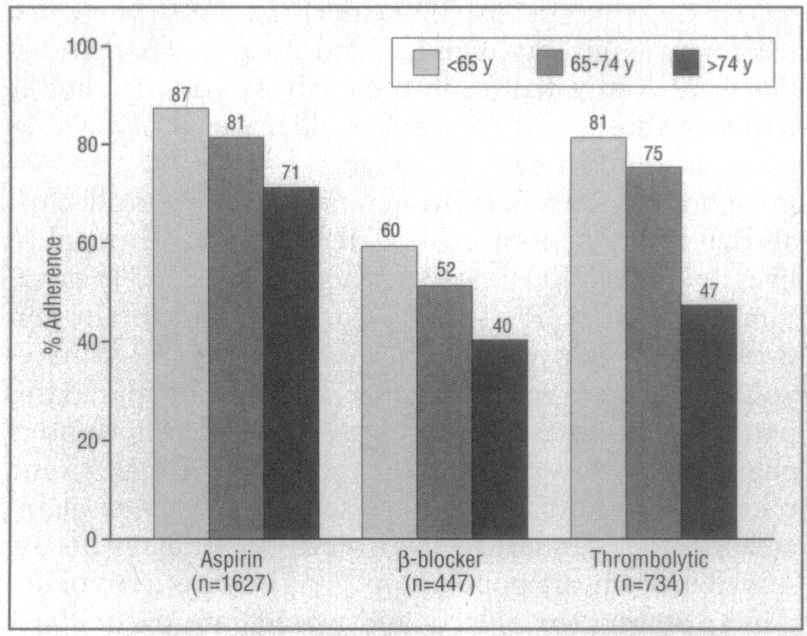

Figure 2. Guideline adherence among eligible patients by age category. ment guidelines among elderly and nonelderly eligible patients in community settings. By extensive review of nearly 2500 medical records of patients presenting with suspected AMI and with clinical and ECG evidence of AMI, we were able to overcome the limitations of prior reports based solely on information recorded in claims or myocardial infarction registry data. Two recent studies ${ }^{15,16}$ of AMI treatment patterns among the elderly reported use of thrombolytic agents and other AMI drug treatments; one of these reports related drug use to shortterm mortality although sample sizes were very small and patients were clustered within six hospitals. Because nonelderly patients with AMI represent approximately one half of all heart attack victims, ${ }^{19}$ we included this important patient population in this study.

We found that among all patients with AMI, eligibility for treatment ranged from $68 \%$ for aspirin use, $38 \%$ for lidocaine use, and $30 \%$ for thrombolytic use to $19 \%$ for $\beta$-blocker use. When relative medical contraindications were dropped as reasons for ineligibility, $96 \%$ of all patients with AMI were eligible to receive aspirin; $33 \%$ were eligible to receive thrombolytic agents; and $24 \%$ were eligible to receive $\beta$-blockers. Among 2409 patients, only 398 had a preexisting disease or history contraindicating use of thrombolytic agents; of those without such contraindications, 620 presented beyond 12 hours after onset of symptoms. Another 598 presented within 12 hours of onset of symptoms but did not have ST-segment elevations on ECG.

The observation that only 447 (19\%) patients were eligible to receive $\beta$-blockers is conservative. Since the main reasons for exclusion from $\beta$-blocker eligibility were sustained bradyarrhythmia and hypotension at hospital presentation, it is likely that a number of patients experienced these conditions for only a limited period, thus becoming eligible to receive $\beta$-blockers subsequently.

Among populations eligible for effective, lifesaving drugs, we observed moderately high rates of use of aspirin $(81 \%)$, moderate use of thrombolytic agents ( $72 \%$ ), and low use of $\beta$-blockers (53\%). Twenty percent of patients without indication for lidocaine received this potentially harmful agent. Although previous studies ${ }^{11-13}$ have examined utilization rates of aspi-

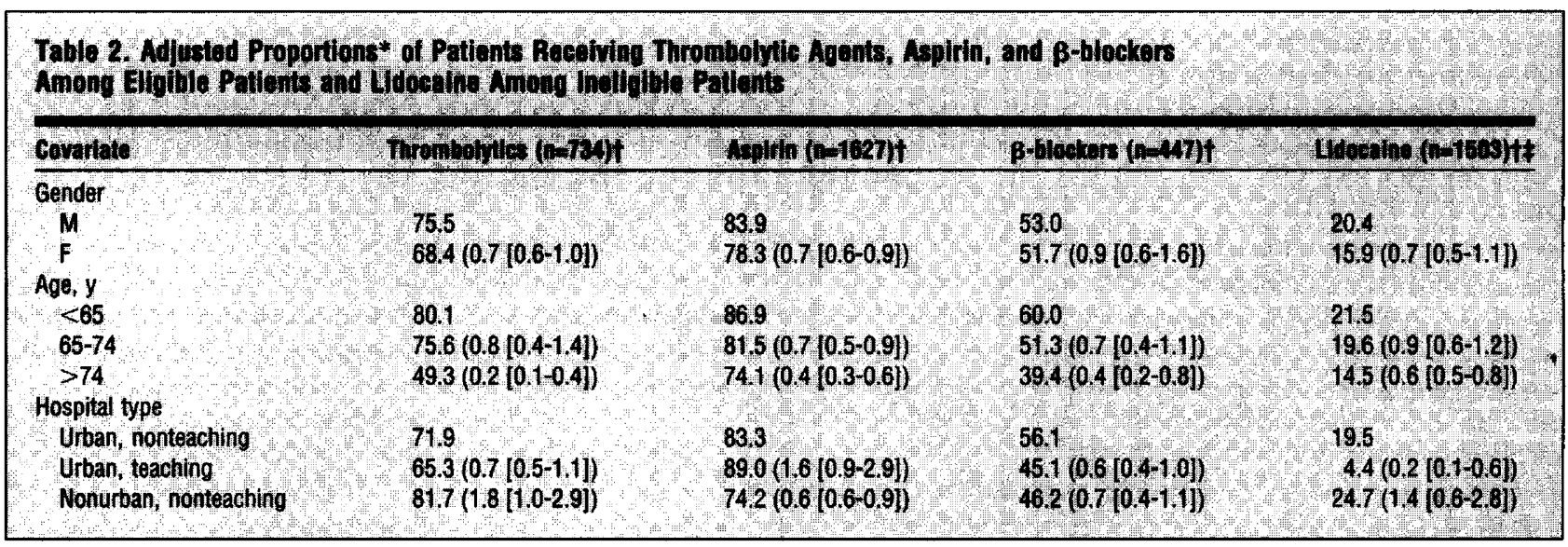

* Predicted probabilities based on Liang and Zeger ${ }^{18}$ logistic regression analyses, controlling for all other characteristics in model. Figures in parentheses represent odds ratios [95\% confidence intervals].

tReferent group for odds ratio for age is under $65 \mathrm{y}$; for gender, males; and for hospital type, urban, nonteaching hospital.

fFor lidocaine, we measured noncompliance as the proportion of patients without indications receiving this agent. 


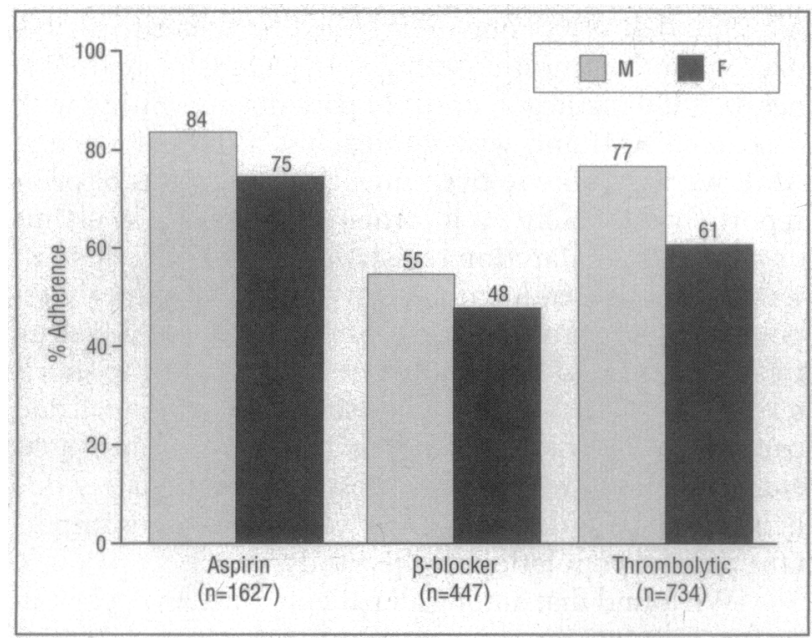

Figure 3. Guideline adherence among eligible patients by gender.

rin, $\beta$-blockers, lidocaine, and thrombolytic agents among total populations of patients with AMI, data limitations in those investigations precluded investigators from defining absolute or relative contraindications and so establish appropriateness of therapy. Failure to define drugspecific eligible populations may lead to biased conclusions (eg, that treatments like thrombolytic agents are used in only a "minority of AMI patients,"20 or among very small fractions of the elderly).

After controlling for the presence of all indications and contraindications, we found that, on average, adherence to guidelines by the American College of Cardiology and the American Heart Assocation during the study period was higher (63\%) than reported in previous studies even for thrombolytic agents in those older than 65 years. A recent study of more than 65000 elderly patients with first heart attacks between 1988 and 1992 found that thrombolytic use increased from $11 \%$ to $18 \%$ from 1988 to 1990 , and plateaued at this level through 1992; the gross utilization rate among elderly patients in this previous study was approximately $20 \%$ for those aged 65 to 69 years and approximately $15 \%$ for those aged 70 to 74 years. In our study, similar proportions of total (eligible plus ineligible) elderly patients with AMI received thrombolytic agents (28\%), but $63 \%$ of eligible elderly patients were treated with these agents. These discrepancies underline the need to define treatment eligibility to measure meaningful utilization rates.

Results that we report for thrombolytic use among eligible elderly patients were not significantly influenced by the use of emergency revascularization procedures. When angioplasty and coronary artery bypass surgery occurring within 24 hours of admission were included in the analysis, only a small change in overall adherence rates $(<3 \%)$ was observed. Do-not-resuscitate orders did not significantly affect treatment adherence rates for use of thrombolytic agents (or other drugs). For example, of 332 patients with do-not-resuscitate orders, only three were eligible to receive thrombolytic agents, and one was eligible to receive $\beta$-blocker therapy.

We observed consistent relationships between patient characteristics, especially age and gender, and guideline adherence. Prior research has demonstrated that an-

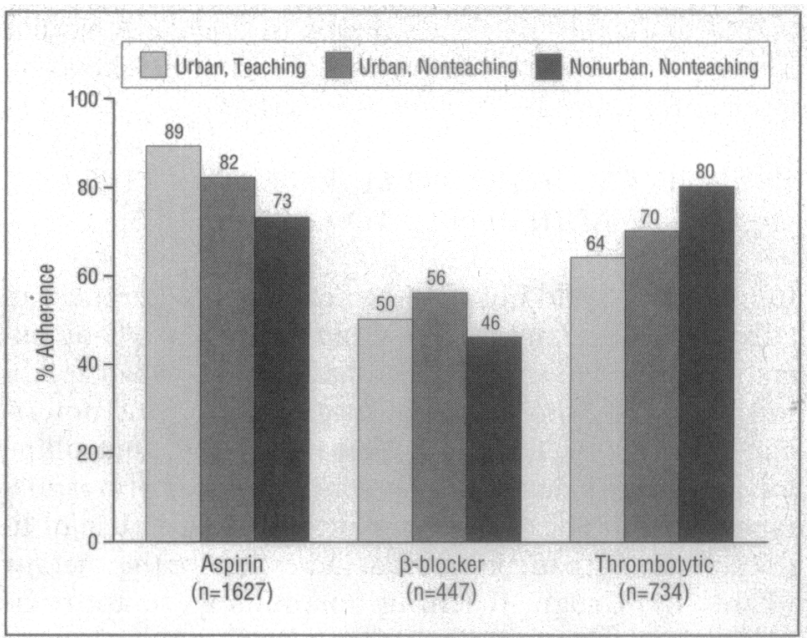

Figure 4. Guideline adherence among eligible patients by hospital type.

giography, coronary artery bypass graft surgery, and percutaneous transluminal coronary angioplasty decline with increasing age; these cardiac procedures were also less common among women than among men. ${ }^{13}$ The present report indicates that reduced levels of use of effective therapies persisted in elderly patients even when treatment eligibility, gender, and hospital characteristics were controlled for in the analysis. Among eligible patients, those aged 75 years or older were only $60 \%$ as likely as younger patients to receive thrombolytic treatment despite the reports from randomized controlled trials that these agents are associated with an additional 10 lives saved per 1000 treated for those aged 65 to 74 years. ${ }^{21}$ Similarly, the OR of administration of aspirin and $\beta$-blocker therapy among those aged 65 to 74 years vs younger patients was 0.67 and 0.70 , respectively, and this pattern of underuse of effective AMI treatments among eligible patients was even more pronounced in the 75 and older age category. Female patients were also at increased risk of undertreatment, corroborating results observed in Medicare populations. ${ }^{11,13}$ These patterns of underuse among the elderly and women are particularly intriguing since these populations have been typically underrepresented in randomized controlled trials.,22

In general, neither a hospital's teaching status nor its location (urban vs nonurban) influenced utilization rates of the study drugs. However, clinicians in urban teaching hospitals were less likely to prescribe lidocaine inappropriately than in urban nonteaching hospitals, and aspirin use was lower in nonurban areas.

Although use of lifesaving therapies for eligible patients with AMI is higher than previously reported, further improvements in guideline adherence rates, especially among elderly people and women, could lead to even further improvement in survival and morbidity for this major disease. Innovative clinician education and systems improvements, aimed at increasing use of effective agents, need to be developed and tested in combination with public education aimed at early recognition of AMI. Government and private health care programs could then be encouraged to adopt the most cost-effective initiatives that can reduce mortality and morbidity resulting from this major disease. 
Accepted for publication November 22, 1995.

From the Department of Ambulatory Care and Prevention, Harvard Medical School and Harvard Pilgrim Health Care, Boston, Mass (Drs McLaughlin and Soumerai and Ms Casey); Departments of Health Policy and Management (Dr Willison) and Biostatistics (Dr Cheng), Harvard School of Public Health, Boston; Gerontology Division (Dr Gurwitz) and Cardiovascular Division (Drs Hauptman and Antman), Department of Medicine, Brigham and Women's Hospital, Harvard Medical School; Healthcare Education and Research Foundation, St Paul, Minn (Dr Borbas and Mss McLaughlin and Morris); Department of Health Care Policy, Harvard Medical School (Dr Guadagnoli); Hennepin County Medical Center, Minneapolis, Minn (Dr Asinger); and The Minnesota Heart Institute, Minneapolis (Dr Gobel).

This work was supported in part by grant HSO 7357-03 from the Agency for Health Care Policy and Research, Harvard Medical School, and the Harvard Pilgrim Health Care Foundation, Boston.

We are indebted to the participating hospitals in the Minnesota Clinical Comparison and Assessment Program for facilitating access to the medical records of study patients; to Suzanne Fletcher, MD, for review of the final manuscript; to Xiaoming Gao, MS, for statistical support; to Ann Payson, MA, for the preparation of the manuscript and illustrations; to Sharon Hawley for assistance in coding of the ECGs; and to the Harvard AMI Patient Outcomes Research Team for comments on preliminary data.

Reprint requests to Department of Ambulatory Care and Prevention, Harvard Medical School and Harvard Pilgrim Health Care, 126 Brookline Ave, Suite 200, Boston, MA 02215 (Dr T. J. McLaughlin).

\section{RIIIRIVIS}

1. Goldman L, Cook EF. The decline in ischemic heart disease mortality rates: an analysis of the comparative effects of medical interventions and changes in lifestyle. Ann Intern Med. 1984;101:825-836.

2. Yusuf $S$, Peto $R$, Lewis $J$, et al. Beta blockade during and after myocardial infarction: an overview of the randomized trials. Prog Cardiovasc Dis. 1985; 17:335-371.

3. Yusuf S, Sleight P, Held P, McMahon S. Routine medical management of acute myocardial infarction: lessons from overviews of recent randomized controlled trials. Circulation. 1990;82(suppl 2):117-134.

4. ISIS-1 (First International Study of Infarct Survival). Collaborative Group: randomized trial of intravenous atenolol among 16,027 cases of suspected acute myocardial infarction: ISIS-I. Lancet. 1986;2:57-65.

5. ISIS-2 (Second International Study of Infarct Survival). Collaborative Group: randomised trial of intravenous streptokinase, oral aspirin, both or neither among
17,187 cases of suspected acute myocardial infarction: ISIS-II. Lancet. 1988; 2:349-360

6. Gurwitz JH, Goldberg RJ, Gore JM. Coronary thrombolysis for the elderly. JAMA. 1991;265:1720-1723.

7. Jansen RWMM, Gurwitz JH. Controversies surrounding the use of betablockers in older patients with cardiovascular disease. Drugs Aging. 1994;4: 175-183.

8. Hine LK, Laird N, Hewitt $P$, Chalmers $T$. Meta-analytic evidence against prophylactic use of lidocaine in acute myocardial infarction. Arch intern Med. 1989; 149:2694-2698.

9. ACC/AHA Task Force. Guidelines for the early management of patients with acute myocardial infarction: a report of the American College of Cardiology/ American Heart Association Task Force on Assessment of Diagnostic and Therapeutic Cardiovascular Procedures (Subcommittee to Develop Guidelines for the Early Management of Patients With Acute Myocardial Infarction). J Am Coll Cardiol. 1990;16:249-292.

10. Soumerai SB, McLaughlin TJ, Avorn JA. Improving drug prescribing in primary care: a critical analysis of the experimental literature. Milbank 0.1989 ; 67:268-317.

11. Pashos CL, Normand SLT, Gartinkle JB, et al. Trends in the use of drug therapies in patients with acute myocardial infarction: 1988 to 1992. J Am Coll Cardiol. 1994;23:1023-1030.

12. Ayanian J, Hauptman PJ, Guadagnoli $E$, et al. Knowledge and practices of generalist and specialist physicians regarding drug therapy for acute myocardial infarction. N Engl J Med, 1994;331:1136-1142.

13. Udvarhelyi IS, Gatsonis C, Epstein AM, Pashos CL, Newhouse JP, McNeil BJ Acute myocardial infarction in the Medicare population: process of care and clinical outcomes. JAMA. 1992;268:2530-2536.

14. Rogers WJ, Bowlby LJ, Chandra NC, et al. Treatment of myocardial infarction in the United States (1990 to 1993): observations from the National Registry of Myocardial Infarction. Circulation. 1994:90:2103-2114.

15. Meehan TP, Hennen J, Radford MJ, et al. Process and outcome of care for acute myocardial infarction among Medicare beneficiaries in Connecticut: a quality improvement demonstration project. Ann Intern Med. 1995;122:928936.

16. Ellerbeck EF, Jencks SF, Radford MJ, et al. Quality of care for Medicare patients with acute myocardial infarction: four-state pilot study from the Cooperative Cardiovascular Project. JAMA. 1995;273:1509-1514.

17. Borbas C, McLaughlin D, Schultz A. Minnesota Clinical Comparison and Assessment Program: bridging the gap between clinical guidelines and clinical practice. In: Coors B, ed. Bridging the Gap Between Theory and Practice Guidelines. St Paul, Minn: Hospital Research and Educational Trust; 1993:37-77.

18. Liang KY, Zeger SL. Longitudinal data analysis using generalized linear models. Biometrika. 1986;73:13-22.

19. National Heart, Lung, and Blood Institute. Morbidity and Mortality: Chartbook on Cardiovascular, Lung, and Blood Diseases. Bethesda, Md: US Dept of Health and Human Services, Public Health Service, National Institutes of Health; January 1992.

20. National Heart, Lung, and Blood Institute. Emergency Department: Rapid Identification and Treatment of Patients With Acute Myocardial Infarction. Bethesda, Md: US Dept of Health and Human Services, Public Health Service, National Institutes of Health; September 1993.

21. Fibrinolytic Therapy Trialists' Collaborative Group. Indications for fibrinolytic therapy in suspected acute myocardial infarction: collaborative overview of early mortality and major morbidity results from all randomized trials of more than 1000 patients. Lancet. 1994;343:311-322.

22. Gurwitz JH, Col NF, Avorn J. The exclusion of the elderly and women from clinical trials in acute myocardial infarction. JAMA. 1992;268:1460-1467. 